\title{
1 Regulating innovative health technologies: dialectics, dialogics, and the 2 case of faecal microbiota transplants
}

3 Final pre-submission manuscript

4 Published in: (2020) Journal of Law, Innovation and Technology doi:

$5 \quad 10.1080 / 17579961.2020 .1815403$

6 Jenny Kaldor, ${ }^{1 *}$ Lisa Eckstein, ${ }^{1}$ Dianne Nicol, ${ }^{1}$ Cameron Stewart ${ }^{2}$

$7 \quad{ }^{1}$ Faculty of Law, The University of Tasmania, Hobart, Australia

$8 \quad{ }^{2}$ Faculty of Law, The University of Sydney, Sydney, Australia

$10 *$ Corresponding author email: Jenny.Kaldor@utas.edu.au

11 Short biographical notes:

12 Jenny Kaldor is a Postdoctoral Research Fellow in the Faculty of Law, the University of

13 Tasmania. Her research focuses on regulatory and policy innovation in medicine and public

14 health. ORCID 0000-0002-1299-6023.

15 Lisa Eckstein is a Senior Lecturer in Law and Medicine in the Faculty of Law, the University

16 of Tasmania. Her area of specialisation is the ethical and legal implications of genetic and other

17 medical research. Before joining the University of Tasmania, Lisa completed her Masters of

18 Health Law at the University of Sydney, a Doctor of Juridical Science at Georgetown

19 University Law Centre and a post-doctoral fellowship in the Department of Bioethics at the

20 National Institutes of Health. ORCID 0000-0002-7161-7521.

21 Dianne Nicol is a Professor of Law in the Faculty of Law, and Director of the Centre for Law

22 and Genetics, the University of Tasmania. Her current research focuses primarily on the

23 regulation of personalized medicine, biobanking, genome editing and other emerging

24 technologies, together with commercialisation of biotechnology and patenting of

25 biotechnological inventions. ORCID 0000-0002-6553-2839.

26 Cameron Stewart is a Professor of Health, Law and Ethics at Sydney Law School, the

27 University of Sydney. He is a member of Sydney Health Law and an associate of Sydney Health

28 Ethics, Sydney Medical School. He has degrees in economics, law and jurisprudence. ORCID 
29 0000-0003-3625-0899.

30

31 Words: 4812

32 


\section{Regulating innovative health technologies: dialectics, dialogics, and the} case of faecal microbiota transplants

This paper interrogates the common characterisation of innovative health technologies "leading", while law and regulation "lag" behind. We analysed the case of faecal microbiota transplants (FMT), an innovative procedure whose regulatory status remains in flux worldwide. We searched the literature for papers that described the regulation of FMT, and coded these according to a simple analytic framework. We identified 21 relevant papers. To date, no jurisdiction has implemented FMT-specific regulation. Instead, FMT is dealt with under a range of approaches, which include fitting it within existing regulation, and the use of "soft" law. We found that metaphor, or argument by analogy, played a central role in delineating the potential regulatory options. We also found the relationship between innovation and regulation to be more dialogue than race. These findings are important because they suggest a less oppositional starting point in discussions of how innovative health technologies should be regulated.

Keywords: regulation; innovation; health technology; faecal microbiota transplant

\section{Introduction}

The relationship between innovative health technologies and the law is often characterised as one in which technology leads, while the law is "outpaced", "lagging" or "limping" behind. ${ }^{1}$ Innovation in health, as in other domains, is seen as a fluid, fast-

\footnotetext{
${ }^{1}$ Lyria Bennett Moses, 'Understanding Legal Responses to Technological Change: The
} Example of in Vitro Fertilization' (2005) 6 Minnesota Journal of Law, Science \& Technology 505, 516; Lyria Bennett Moses, “Agents of Change: How the Law “Copes” with Technological Change' (2011) 20 Griffith Law Review 763, 764; Dianne Nicol and others, 
paced process, dependent on flexible, open-ended thinking, and a tolerance for risk and uncertainty. Laws and other forms of regulation, by contrast, are often regarded as static systems, slow to adapt and rigid once in place. In this characterisation, the goals of regulation - protecting patient safety, ensuring access to effective therapies, controlling public spending on healthcare, and promoting the ethical conduct of researchers and clinicians - can appear to frustrate the translational goals of innovative health research and development. This kind of relationship can be described as dialectical: regulation is seen to oppose or block innovation; then, in the struggle of competing forces, new forms of regulation emerge, only to be later challenged by further innovations. This dialectical dynamic - opposition, followed by synthesis and then further opposition - has previously played out in the context of innovations such as IVF, human gene therapy, autologous stem cell therapies and embryonic stem cell research. It is currently manifest in debates around precision medicine technologies such as genome editing using the CRISPR-Cas9 system, drug/diagnostic pairs, and 3D bioprinting. This dynamic is important because it shapes and constrains the range of regulatory responses which are deemed possible, in response to innovation. In the 2014-16 debates surrounding the UK's Medical Innovation Bill, for example, the bill's proponent Lord Saatchi argued that the common law was stifling medical innovation by imposing too stringent a test of negligent liability on doctors. ${ }^{2}$ He proposed that a legal exemption be created for doctors so they would face no liability in negligence for performing

'Precision Medicine: Drowning in a Regulatory Soup?' (2016) 3 Journal of Law and the Biosciences 281.

${ }^{2}$ Medical Innovation Bill 2014 (UK). 
innovative therapies, under certain conditions. ${ }^{3}$ While the bill ultimately failed, discourse surrounding it exemplified the belief that regulation and innovation are diametrically opposed to one another; it was argued that the laws had a negative effect on medicine as they could not accommodate innovation. ${ }^{4}$

The premise of this paper is that the relationship between innovative health technologies and the law should not be presumed to be dialectical, but is rather a matter for empirical investigation in each new case. We use the case study of faecal microbiota transplantation to put this the relationship between innovation and regulation under the microscope. Is there a lag between the two? What is the reason for the lag? Through what kinds of strategies do laws and other regulation seek to "catch up" with technology? And how could these be improved? To what degree do the forms of regulation contribute to or guide the emergence of innovation?

We begin by defining the key concepts, "innovative health technology" and "laws and other forms of regulation". We then explain how faecal microbiota transplantation fits our criteria for an innovative health technology, and how it is currently regulated. Finally, we outline our approach to the case study, which uses qualitative content analysis of scholarly papers, and set out our findings. This study leads us to suggest that, in this case at least, the encounter between innovative health technologies and the law is more dialogic than dialectic: more a dialogue than a race.

\footnotetext{
${ }^{3}$ Ibid s 1 .

${ }^{4}$ Bernadette Richards and others, 'The Medical Innovation Bill: Still More Harm than Good' (2015) 10 Clinical Ethics 1. Instead of the Bill, the UK Parliament passed the Access to Medical Treatments (Innovation) Act 2016 (UK), which allowed for the creation of a register of innovative treatments.
} 
94 We found that regulatory pathways were determined at key decision-points, one of the

95 most important of which was the choice of analogy to describe regulatory interactions.

96 That is: based on key characteristics, which existing technology, or technologies, does

97 the new technology resemble? We conclude by noting some implications of our

98 findings for the regulation of innovative health technologies more broadly.

\section{2. Framing the case study: key context and concepts}

\section{0 (a) What are innovative health technologies?}

101 All health technologies involve "the application of organized knowledge and skills in

102 the form of devices, medicines, vaccines, procedures and systems developed to solve a

103 health problem and improve quality of lives". ${ }^{5}$ We define innovative health technologies

104 as having two additional characteristics. First, they are radically new. They represent a

105 paradigm shift, and “open up new possibilities and allow us to do things that haven't

106 been done before," as opposed to being merely "a variation or extension or

107 improvement on something we can already do". ${ }^{6}$ Secondly, they are health technologies

108 at an early or investigational stage of their life cycle, with their full range of

109 applications not yet fully understood. They are "still emergent... and have not yielded

110 many applications and societal consequences". ${ }^{7}$

${ }^{5}$ World Health Assembly, Resolution on health technologies, WHA60.29, 60 $0^{\text {th }}$ World Health Assembly, 2007; available online at https://www.who.int/healthsystems/WHA60_29.pdf?ua=1 (accessed March 2019).

${ }^{6}$ David Hunter, 'How to Object to Radically New Technologies on the Basis of Justice: The Case of Synthetic Biology' (2013) 27 Bioethics 426.

${ }^{7}$ Philip AE Brey, 'Anticipatory Ethics for Emerging Technologies’ (2012) 6 NanoEthics 1, 1. 


\section{1 (b) What is law and other regulation?}

112 Over the past three decades, the field of regulatory scholarship has significantly

113 reshaped our understanding of regulation, both in its meaning and in its scope. In

114 particular, regulation is no longer considered the exclusive purview of the State and its

115 laws: though these remain important, regulation today is widely described as

116 "decentred". ${ }^{8}$ What this means is that regulation is no longer top-down and linear, but

117 rather encompasses a wide array of actors and activities, interacting within "regulatory

118 space". ${ }^{9}$ Regulation can take the form of formal, direct mechanisms - e.g. statutes,

119 regulations, policies - as well as less formal, and less direct mechanisms - e.g. industry

120 self-regulation, stakeholder forums, funding decisions. In this paper, we adopt Black's

121 definition of regulation, which is that "regulation, or regulatory governance, is the

122 organised attempt to manage risks or behaviour in order to achieve a publicly stated

123 objective or set of objectives". ${ }^{10}$ Following Morgan and Yeung, we use the expression

124 "laws and other forms of regulation", or "law and regulation", to emphasise that, as

${ }^{8}$ Julia Black, 'Decentring Regulation: Understanding the Role of Regulation and SelfRegulation in a "Post-Regulatory" World' (2001) 54 Current legal problems 103. See also Bronwen Morgan and Karen Yeung, An Introduction to Law and Regulation: Text and Materials (Cambridge University Press 2007) 4.

${ }^{9}$ Leigh Hancher and Michael Moran, 'Organizing Regulatory Space’ in Robert Baldwin, Colin Scott and Christopher Hood (eds), A Reader on Regulation (Oxford University Press 1998); Susan MC Gibbons, 'Mapping the Regulatory Space' in Andrew Smart (ed), Jane Kaye and others, Governing Biobanks: Understanding the Interplay between Law and Practice (Hart Pub 2012).

${ }^{10}$ Julia Black, 'Learning from Regulatory Disasters' (2014) 10 Policy Quarterly 3. 
125 legal scholars, we bring a legal perspective to this expanded view of regulation. ${ }^{11}$ In the

126 context of innovations in health care, we are particularly interested in the impact of

127 therapeutic goods regulation, professional ethics and discipline, tort laws, healthcare

128 complaints, and intellectual property laws, but we recognise that these well-mapped

129 areas of legal inquiry have roots that grow out into less well known areas of normative

130 control.

\section{3. Qualitative content analysis}

\section{2 (a) FMT as a case study}

133 We selected the case study of faecal microbiota transplantation $(\boldsymbol{F M T})$ in order to

134 investigate the ways in which innovative health technologies encounter law and

135 regulation. Described by one of its pioneers as a therapeutic strategy "at the fringes of

136 medicine", ${ }^{12}$ FMT "consists of the infusion of faeces from a healthy donor to the

137 gastrointestinal $(\boldsymbol{G I})$ tract of a recipient patient, in order to treat a specific disease

138 associated with alteration of gut microbiota". ${ }^{13}$ Although its mode of action is not yet

139 completely understood, broadly speaking, FMT treats disease by "restoring the

140 phylogenetic diversity and microbiota more typical of a healthy person". ${ }^{14}$ There is now

141 a robust body of evidence to support the efficacy of FMT as a treatment for recurrent

\footnotetext{
${ }^{11}$ Morgan and Yeung (n 8) 14.

12 Thomas J Borody and Alexander Khoruts, 'Fecal Microbiota Transplantation and Emerging Applications’ (2012) 9 Nature Reviews Gastroenterology \& Hepatology 88, 91.

${ }^{13}$ Giovanni Cammarota and others, 'European Consensus Conference on Faecal Microbiota Transplantation in Clinical Practice’ (2017) 66 Gut 569, 569.

${ }^{14}$ Colleen R Kelly and others, 'Update on Fecal Microbiota Transplantation 2015: Indications, Methodologies, Mechanisms, and Outlook’ (2015) 149 Gastroenterology 223, 223.
} 
142 Clostridium difficile infection (rCDI) ${ }^{15}$ an "aggressive intestinal superbug"16 in relation

143 to which antibiotic resistance is a growing concern. In addition, FMT is under

144 investigation for a number of other GI conditions, as well as some non-GI diseases such 145 as diabetes.

146 In both the rCDI indication, and other areas of investigation, FMT satisfies our

147 criteria for an innovative health technology. While the medicinal use of faeces has been 148 documented as far back as fourth-century China, ${ }^{17}$ FMT in its modern form has been 149 used sporadically over the past 50 years, gaining pace in the past decade. ${ }^{18}$ However, it

150 fits our criteria of being "radically new" in the sense that it represents a paradigm shift

151 from the "germ theory" based therapies, such as antibiotics, which constituted the

152 previous standard of care for $\mathrm{rCDI} .{ }^{19}$ Instead, FMT engages with the gut microbiota as a

${ }^{15}$ National Institute for Health and Care Excellence (2014) "Faecal microbiota transplant for recurrent Clostridium difficile infection" (IPG485). Available online at https://www.nice.org.uk/guidance/ipg485 (accessed March 2019); Cammarota and others (n 13); Benjamin H Mullish and others, 'The Use of Faecal Microbiota Transplant as Treatment for Recurrent or Refractory Clostridium Difficile Infection and Other Potential Indications: Joint British Society of Gastroenterology (BSG) and Healthcare Infection Society (HIS) Guidelines' (2018) 67 Gut 1920.

${ }^{16}$ Rachel E Sachs and Carolyn A Edelstein, 'Ensuring the Safe and Effective FDA Regulation of Fecal Microbiota Transplantation' (2015) 2 Journal of Law and the Biosciences 396.

${ }^{17}$ Kenneth A Young, 'Of Poops and Parasites: Unethical FDA Overregulation' (2014) 69 Food and Drug Law Journal 555.

${ }^{18}$ Borody and Khoruts (n 12), Cammarota (n 13).

${ }^{19}$ Alexander Khoruts and Michael J Sadowsky, 'Understanding the Mechanisms of Faecal Microbiota Transplantation’ (2016) 13 Nature Reviews Gastroenterology \& Hepatology 508. 
153 "true organ", one that is "integral to human physiology," 20 opening up completely new

154 treatment possibilities. It should be noted that, even in the rCDI indication - i.e., where

155 FMT is best understood - scientists have yet to characterise the precise interactions

156 between the human microbiome and disease and the resultant opportunities and

157 challenges for therapeutic intervention.

158 Moreover, FMT provides an interesting case study because the question of how

159 it should be regulated is very much still in flux. Around the world, regulation of FMT

160 currently runs the gamut "from non-existing to strictly regulated". ${ }^{21}$ So far, no

161 jurisdiction has introduced FMT-specific legislation. Instead, the following approaches

162 have been documented:

163 - FMT is regulated under the existing framework for biologic medicines (e.g.

$164 \quad$ France);

165 - FMT is regulated under the existing framework for biologic medicines, with some

166 customisation (e.g. Canada, UK, USA);

167 - No existing regulations apply, and no new regulations are enacted, but "soft law"

168 in the form of professional guidelines fills the gaps (e.g. Austria); and

169 - No existing regulations apply, and no new regulations are enacted, leaving gaps

170 and lack of clarity (e.g. Australia, China).

${ }^{20}$ Ibid.

${ }^{21}$ Frederick Verbeke and others, 'Faecal Microbiota Transplantation: A Regulatory Hurdle?' (2017) 17 BMC Gastroenterology 128. 
172 Using the databases MedLine, Web of Science, and HeinOnline, we searched the

173 literature for peer-reviewed publications on FMT, published in or after 2013 (when the

174 first randomised study of FMT in rCDI was published), ${ }^{22}$ and which included

175 substantial discussion of legal and regulatory considerations. We used the search terms

$176 f(a)$ ecal microbio* transplant* AND [law* OR regulat* OR polic* OR legislat*]. We

177 identified 21 papers that met our inclusion criteria. Most of these $(n=14)$ were published

178 in scientific journals; 5 were published in law journals and two in health policy

179 journals. ${ }^{23}$ Eleven papers discussed the USA regulatory context, six related to Europe or

180 individual European countries, there was one paper each on Australia and Canada, and

181 two that compared international approaches.

182 Using qualitative content analysis methodology, ${ }^{24}$ we developed a simple coding

183 framework and extracted data in relation to the following three themes:

184 - In relation to FMT, how do commentators characterise the goals of regulation?

185 - What specific regulatory challenges does FMT present?

186 - How could the regulation of FMT be improved?

${ }^{22}$ Els van Nood and others, 'Duodenal Infusion of Donor Feces for Recurrent Clostridium Difficile' (2013) 368 New England Journal of Medicine 407.

${ }^{23}$ As Lewis notes in her 2018 law review article, there has been little discussion of FMT in the legal literature: Myrisha S Lewis, 'Halted Innovation: The Expansion of Federal Jurisdiction over Medicine and the Human Body’ [2018] Utah Law Review 1073.

${ }^{24}$ Margrit Schreier, Qualitative Content Analysis in Practice (Sage Publications 2012). 
(i) What are the goals of regulation in relation to FMT?

189 Beyond the general justifications for regulating health technologies, what should

190 regulation seek to achieve specifically in relation to FMT? In this regard, most

191 commentators drew a distinction between FMT for its established indication of rCDI, as

192 compared with other clinical indications. ${ }^{25}$ For rCDI, commentators expressed a strong

193 concern that regulation might inappropriately hinder access to a very effective treatment

194 (one paper described this course of action as "inhumane"), ${ }^{26}$ while also stressing the

195 importance of maintaining safety, e.g., through rigorous screening of donors and

196 donated stool. For indications other than rCDI, which are still investigational, most

197 commentators focused on the importance of encouraging responsible research and

198 development, rather than patient access.

(ii) What regulatory challenges does FMT present?

200 Three key features of FMT were identified as creating specific regulatory challenges:

201 the nature of the substance itself; the multiple different modes of administration

202 available; and uncertainty regarding the longer-term effects of FMT. First, in terms of

203 the nature of the substance, Sachs and Edelstein note that stool is "unique for the

204 difficulty of its characterisation and the simplicity of its production, and each of these

205 characteristics raises special safety concerns". ${ }^{27}$ Unlike a conventional medicine, it is

${ }^{25}$ See for example, Carolyn A Edelstein and others, 'The Regulation of Fecal Microbiota for Transplantation: An International Perspective for Policy and Public Health’ (2015) 32

Clinical Research and Regulatory Affairs 99, 105.

${ }^{26}$ Verbeke and others (n 21) 6.

${ }^{27}$ Sachs and Edelstein (n 16) 406. 
easy to manufacture and thus obtain a "batch" of product, raising the possibility of DIY

207 procedures, carried out outside the auspices of medicine. Yet, also unlike a conventional

208 medicine, each "batch" - even from the same person - can differ greatly, which

209 presents challenges in its characterisation and therefore for quality control. ${ }^{28}$ This

210 difficulty is encapsulated by the fact that almost all commentators raised the spectre of

211 potentially risky "YouTube FMT" - patients self-administering the procedure on the

212 guidance of online videos.

213 Secondly, there are multiple different ways to carry out an FMT procedure.

214 Stool may come from a known or an unknown donor, or may be autologous, or

215 synthetic; it may be fresh or frozen; it may be administered via enema, colonoscopy, or

216 other means. This gives rise to multiple variables that may influence the efficacy,

217 safety, and acceptability of FMT, and these have not yet been fully disentangled through

218 clinical trials. While guidelines have been published in several jurisdictions, these have

219 tended to be based on expert opinion, due to the lack of robust evidence. ${ }^{29}$ Even in

220 rCDI, most of the available evidence for FMT comes from retrospective case series or

221 systematic reviews, ${ }^{30}$ rather than the "gold standard" of randomised controlled trials.

222 This informs the third feature, which is that little is known about the long-term safety

${ }^{28}$ Diane Hoffmann and others, 'Improving Regulation of Microbiota Transplants' (2017) 358

Science 1390. See also Samuel P Costello and Robert V Bryant, 'Faecal Microbiota

Transplantation in Australia: Bogged down in Regulatory Uncertainty' (2019) 49 Internal Medicine Journal 148, 149.

${ }^{29}$ Cammarota and others (n 13).

${ }^{30}$ SD Goldenberg, 'Faecal Microbiota Transplantation for Recurrent Clostridium Difficile Infection and Beyond: Risks and Regulation' (2016) 92 Journal of Hospital Infection 115. 
223 profile of FMT. In particular, the risks of transmitting communicable diseases, ${ }^{31}$ as well

224 as non-communicable conditions including psychological illnesses, ${ }^{32}$ from the donor to 225 the patient, remain poorly understood.

226 (iii) How could the regulation of FMT be improved?

227 Commentators suggested a variety of alternatives to the current regulation of FMT, in

228 order to better achieve the identified goals (set out at 3.1) and address the identified

229 challenges (set out at 3.2). The regulatory approach advocated by each commentator

230 reflected the priority they gave to these goals and challenges.

231 Several commentators suggested designating a "special status" for FMT, similar

232 to that of blood. ${ }^{33}$ This was based on a view that the rigorous manufacturing standards

233 applied to drugs would be unduly burdensome for an innately variable substance such as

234 human stool. ${ }^{34}$ The blood framework was also considered well suited to addressing the

235 communicable disease risks associated with FMT. ${ }^{35}$ Another suggested option was to

236 classify FMT as a gene-, cell-, or tissue-like product for regulatory purposes. This was

${ }^{31}$ N Kapel and others, 'Practical Implementation of Faecal Transplantation' (2014) 20 Clinical Microbiology and Infection 1098, 1099.

${ }^{32}$ Tim Spector and Rob Knight, 'Faecal Transplants Still Need Good Long Term Trials and Monitoring (Editorial)'.

${ }^{33}$ JC Lagier, 'Faecal Microbiota Transplantation: From Practice to Legislation before Considering Industrialization’ (2014) 20 Clinical Microbiology and Infection 1112; Edelstein and others (n 25).

${ }^{34}$ Lagier (n 33); Mark B Smith, Colleen Kelly and Eric J Alm, 'Policy: How to Regulate Faecal Transplants' (2014) 506 Nature 290.

${ }^{35}$ Smith, Kelly and Alm (n 34). 
237 proposed as a means of addressing FMT risks that fall beyond the risks applicable to

238 blood, including pre-existing risk management frameworks and clear criteria for clinical

239 trial exemptions. ${ }^{36}$ However, in their argument against regulating FMT as a tissue,

240 Megerlin and colleagues stressed the currently limited understanding of its mechanism

241 of action, likened the healthy donor to a "bioreactor", and highlighted the substance's

242 topographical rather than cellular origin. ${ }^{37}$ Based on these distinctions, Megerlin and

243 colleagues argued that the appropriate regulatory paradigm for FMT was as a sui

244 generis biological drug, rather than human tissue. ${ }^{38}$

245 Others suggested that FMT should be regulated "like a drug," but only in certain

246 circumstances. Drawing on the current US system for regulating cord blood, for

247 example, Sachs and Edelstein suggested that while FMT should be regulated as an

248 investigational new drug for its non-approved indications, the cord blood model would

249 be appropriate for the approved indication of rCDI. This model provides differing

250 degrees of oversight based on both the intended recipient (autologous stool and stool

251 donated by close relative, versus banked stool) and the intended use of stool. ${ }^{39}$

252 Responding to the numerous variables in FMT administration, Hoffmann and

253 colleagues proposed a "three-track regulatory system" for the USA, under which the

254 regulation of FMT would differ depending on the product being used, and its context: ${ }^{40}$

\footnotetext{
${ }^{36}$ Verbeke and others (n 21).

${ }^{37}$ F Megerlin and others, 'Faecal Microbiota Transplantation: A Sui Generis Biological Drug, Not a Tissue' (2014) 72 Annales Pharmaceutiques Françaises 217.

${ }^{38}$ Ibid.

${ }^{39}$ Sachs and Edelstein (n 16).

${ }^{40}$ Hoffmann and others (n 28).
} 
(a) When performed by a physician, using known stool, to treat rCDI, FMT would be regulated as the practice of medicine. In indications other than rCDI, FMT would be treated as an investigational drug and regulated accordingly, unless the use meets legal requirements for "clinical innovation".

(b) When performed by a physician, using banked stool, to treat $\mathrm{rCDI}$, the stool bank would be regulated like a tissue bank, with some additional oversight.

(c) "Modified stool-based products" would be regulated like biological drugs.

262 The authors note that the proposed three-track system would not require new legislation,

263 and could be achieved with guidelines. ${ }^{41}$ The importance of guidelines, as opposed to

264 "hard" law, was also notable in the article by Woodworth and colleagues. ${ }^{42}$ This article

265 noted the discrepancy between the US FDA's focus on FMT using stool from a known

266 donor, and the empirical trend towards the use of banked stool. In order to address this,

267 they emphasised the importance of rigorous screening protocols and stakeholder

268 collaboration.

\section{Discussion}

270 By displacing established scientific and social practices, innovative health technologies create uncertainty ${ }^{43}$ and disruption. ${ }^{44}$ As a result, they may not fit - or may appear not to

41 Ibid.

${ }^{42}$ Michael H Woodworth and others, 'Challenges in Fecal Donor Selection and Screening for Fecal Microbiota Transplantation: A Review’ (2017) 8 Gut Microbes 225.

${ }^{43}$ Elen Stokes, 'Demand for Command: Responding to Technological Risks and Scientific Uncertainties’ (2013) 21 Medical Law Review 11.

${ }^{44}$ See for example, Heidi Ledford, 'CRISPR, the Disruptor' (2015) 522 Nature News 20. 
272 fit - into existing legal structures and categories. Innovative health technologies are

273 often deemed, by the public as well as by regulators, to present risks that are

274 qualitatively different from those addressed in existing legislation, and so can prompt

275 calls for new, technology-specific regulations. ${ }^{45}$ Yet, as Stokes has observed, it is rare

276 that a new technology arrives "completely 'lawless"" ${ }^{46}$ Rather, on arrival, innovative

277 health technologies are met by complex webs of existing laws and other forms of

278 regulation: the formal and informal rules which include professional norms and

279 guidelines, legislation, regulation, policy directives, funding conditions, and so on, and

280 which govern existing health technologies.

281 On the basis of our findings regarding FMT, we posit that regulation does not

282 simply react to, or compete with, innovation. While there are many illustrations which

283 support the existence of a dialectical relationship between innovation and regulation, in

284 the case of FMT our findings instead suggest dialogical development. ${ }^{47}$ We use this

285 term to describe the relationship between innovation and regulation as dynamic,

${ }^{45}$ Bert-Jaap Koops, 'Ten Dimensions of Technology Regulation: Finding Your Bearings in the Research Space of an Emerging Discipline' in MEA Goodwin, BJ Koops and RE Leenes (eds), Dimensions of Technology Regulation (Wolf Legal Publishers 2010); Bennett Moses, 'Agents of Change: How the Law "Copes" with Technological Change' (n 1).

${ }^{46}$ Elen Stokes, 'Nanotechnology and the Products of Inherited Regulation' (2012) 39 Journal of Law and Society 93, 94.

${ }^{47}$ The term "dialogic" is often used in relation to Bakhtin's linguistic theories. According to Bakhtin, dialogic development occurs when language changes in response to how it has been used in the past, and it is also shaped by an understanding of how it may be used in the future: see Bakhtin, The Dialogic Imagination: Four Essays (Caryl Emerson ed, Michael Holquist tr, Austin: University of Texas Press 1981). 
relational, open-ended, and engaged in a process of constant iteration. Based on our

287 findings above, we make three observations about the encounter between innovative

288 health technologies and the law. We begin by noting that "innovation" is not, in fact, the

289 object of regulation, and emphasise the importance of specificity. Secondly, we identify

290 the centrality of metaphor in channelling discussion towards one regulatory pathway or

291 another. Finally, we describe the ways in which these metaphors shape the dialogic

292 relationship between innovative health technologies and regulation.

\section{3 (a) Regulating “innovation”}

294 One important observation from our case study of FMT is that, even where an

295 innovative health technology is the object of regulation, "innovation" per se is not the

296 thing being regulated. Rather, the technology's degree of innovation derives from the

297 convergence of other, more specific qualities, and it is these qualities that create any

298 uncertainty, and prompt calls for new or different regulation. So, in the case of FMT,

299 the innovation is the concept of using stool to repopulate the microbiome, as opposed to

300 the previously standard practice of using antibiotics to address the infection. The

301 problem facing scientists, clinicians, and regulators in this space is a specific, rather

302 than a general one: an FMT challenge, not an "innovation" challenge. This underscores

303 the importance of case-by-case, empirical investigation, to determine whether in fact the

304 specific innovative technology confirms to broader narratives about innovation and

305 regulation.

\section{6 (b) The "channelling" role of metaphor}

307 Our second observation relates to the central role played by metaphor, or reasoning by

308 analogy, in discussions of how FMT should be regulated. We noted at the outset that an

309 innovative health technology is paradigm-shifting: by definition, it is not "like" any 
310 predecessor technology. Yet, whether advocating that FMT should be regulated like a

311 biological, like blood, or like an organ, most suggestions sought to fit FMT within an

312 existing regulatory paradigm, based on the notion that it shares qualities with another

313 product or process. As Megerlin and colleagues note, even the term "faecal microbiota

314 transplant" is itself a metaphor, and not necessarily an accurate one: rather, its usage

315 reflects the "lexical disruption" brought about by "scientific revolution" ${ }^{48}$ Our findings

316 suggest that the decision to compare the innovative health technology to an existing

317 technology is in fact an important regulatory moment. In that moment, the innovative

318 technology is "channelled" towards an existing regulatory regime based on a

319 prioritisation of identified goals and challenges, and a process begins.

\section{0 (c) Dialogical development}

321 Our third observation relates to this process, which - at least in the context of FMT - is

322 better described as a dialogue than as a "race" in which law lags behind technology.

323 Across jurisdictions, the emergence of FMT has prompted consideration of whether

324 existing regulation applies or is appropriate; adjustments - including in the form of

325 screening protocols, guidelines, and subsidiary legislation - are then made, which in 326 turn inform future developments. As Sachs and Edelstein acknowledged, even the de

${ }^{48}$ Megerlin and others (n 37) 3. Similarly, the UK's National Institute for Health and Care Excellence (NICE) "recognised that the enteric infusion of donor faeces is not a transplant in the usual sense of transplanting body tissues, but... accepted that faecal microbiota transplant has become an accepted term to describe this procedure": National Institute for Health and Care Excellence (2014) "Faecal microbiota transplant for recurrent Clostridium difficile infection" (IPG485). Available online at https://www.nice.org.uk/guidance/ipg485 (accessed March 2019). 
327 novo legislation option would not be pursed in a vacuum, and "must be considered in

328 light of decisions that [regulators have] already made in this area". ${ }^{49}$ Noting that the US

329 Food and Drug Administration $(\boldsymbol{F D} \boldsymbol{A})$ had redefined its position on FMT three times,

330 Riley and Olle perceive not regulatory lag, but an iterative process: FDA gaining

331 "additional experience with the potential technologies before it wades into more formal

332 rulemaking". ${ }^{50}$ As highlighted in multiple papers, FMT is a quickly evolving therapy

333 area, in which the different indications - and their varying strengths of evidence - are as

334 determinative of regulatory choice as the technology itself. In this context, an iterative

335 or dialogic understanding of regulation may in fact be the most appropriate way to

336 "cope" with innovation. ${ }^{51}$

\section{5. Conclusion}

338 In contrast to the common characterisation of innovative technologies "leading", while

339 law "lags" behind, our case study revealed a less oppositional, more fluid and

340 conversational dynamic. Regulatory dynamics, in this study, were shown to be a process

341 rather than a given; involving incremental adjustments, trial-and-error, and mechanisms

342 other than formal law and regulations. The extent to which these findings can be

343 generalised to other innovative health technologies is beyond the scope of this paper.

344 Certainly, and as we noted at the outset, there are many examples that support the

345 traditional, dialectical framing of the relationship between regulation and innovation.

346 However, our research shows that this is not the only possible relationship, and supports

\footnotetext{
${ }^{49}$ Sachs and Edelstein (n 16) 413.

${ }^{50}$ Margaret F Riley and Bernat Olle, 'FDA's Pathway for Regulation of FMT: Not so Fraught' (2015) 2 Journal of Law and the Biosciences 742.

${ }^{51}$ Bennett Moses, ‘Agents of Change: How the Law “Copes” with Technological Change’ (n 1).
} 
347 an approach which investigates each new regulatory encounter empirically, and on its

348 own merits. Moreover, conceiving of the relationship in dialogic terms has important

349 implications for stakeholders, including scientists, regulators, and the public. In

350 challenging some of the entrenched assumptions about law versus innovation, a dialogic

351 framing may open up possibilities for a more collaborative discussion about how best to

352 regulate specific innovative health technologies.

353

354

355

356

357

358 Funding: This work was supported by the Australian Research Council under ARC

359 Discovery Grant DP180101262. 